\title{
BMJ Open Economic burden of cirrhosis in Catalonia: a population-based analysis
}

\author{
Mireia Miquel, ${ }^{1,2}$ Montserrat Clèries, ${ }^{3}$ Mercedes Vergara, ${ }^{1,2}$ Emili Vela ${ }^{3}$
}

To cite: Miquel $\mathrm{M}$, Clèries $\mathrm{M}$, Vergara $\mathrm{M}$, et al. Economic burden of cirrhosis in Catalonia: a populationbased analysis. BMJ Open 2018;8:e018012. doi:10.1136/ bmjopen-2017-018012

- Prepublication history and additional material for this paper are available online. To view these files, please visit the journal online (http://dx.doi. org/10.1136/bmjopen-2017018012).

Received 5 June 2017 Revised 27 October 2017 Accepted 4 December 2017

D) Check for updates

${ }^{1}$ Hepatology Unit, Digestive Disease Department, Parc Tauli Hospital Universitari, Institut d'Investigació i Innovació Parc Taulí I3PT, Universitat Autònoma de Barcelona, Sabadell, Spain ${ }^{2}$ CIBERHED, Instituto de Salud Carlos III, Madrid, Spain

${ }^{3}$ Unitat d'informació i Coneixement, Servei Català de la Salut, Generalitat de Catalunya, Barcelona, Spain

Correspondence to

Dr Mireia Miquel;

mmiquel@tauli.cat

\begin{abstract}
Background Cirrhosis is a chronic disease with high morbidity and mortality. Few studies have evaluated healthcare resource use in patients with cirrhosis.
\end{abstract}

Objective We aimed to describe the point prevalence of cirrhosis on 31 December 2012 and the population-level distribution of healthcare resource use and expenditures in a non-selected population of patients with cirrhosis, stratified by whether their disease was compensated or decompensated, and by comorbidity burden.

Methods This population study included all known patients aged $>18$ years with cirrhosis (according to International Classification of Diseases, ninth revision) in Catalonia, Spain, on 31 December 2012. We evaluated healthcare resource use and expenditure during 2013, taking into account the presence of decompensation before or during 2012.

Results We documented 34740 patients diagnosed with cirrhosis ( $58.7 \%$ men; mean age $61.8 \pm 14$ years), yielding a point prevalence of 460 per 100000 inhabitants on 31 December 2012. Annual mortality was $9.1 \%$. During 2013, healthcare expenditures on patients with cirrhosis totalled $€ 142.1$ million (€4234 per patient), representing $1.8 \%$ of the total 2013 healthcare budget of Catalonia. Hospitalisation costs accounted for $35.1 \%$ of the total expenditure and outpatient care accounted for $22.4 \%$. MultivariateMultivariate logistic regression identified morbidity burden, HIV infection, hospitalisation and emergency room visits during 2012 as independent predictors of expenditure above the 85th centile (area under the receiver operating curve, $0.88(95 \% \mathrm{Cl} 0.883$ to $0.893, \mathrm{P}<0.001)$ ).

Conclusions Cirrhosis accounts for a high proportion of healthcare resource usage and expenditures; hospitalisation accounted for the highest expenditures.

\section{INTRODUCTION}

Cirrhosis is the late stage in progressive liver disease of varying aetiologies. Not much is known about the prevalence of cirrhosis among older adults but it is expected to increase, in part due to the rising incidence of non-alcoholic fatty liver disease and an ageing hepatitis $\mathrm{C}$ population. ${ }^{1-3}$ In fact, there is an increasing trend of diagnosis of chronic liver disease and its decompensation and complications (including hepatocellular carcinoma). ${ }^{4}$

The time interval between the compensated phase of cirrhosis and the development
Strengths and limitations of this study

- We performed cost analysis using a populationbased database including 34740 patients diagnosed with cirrhosis in Catalonia, thus minimising selection bias.

- The study quantified the total economic impact of cirrhosis in relation to hepatic decompensation and determined the distribution of the costs involved in treating patients with this condition.

- We have not included important information such as the aetiology of cirrhosis and other important clinical data for characterising the study population and the evolution of the disease (ie, MELD, Child-Pugh or ACLF).

- The use of a validated risk assessment tool made it possible to calculate individuals' morbidity burden and to analyse the impact of multimorbidity on resource use and costs.

- Although the database is periodically monitored and has high quality data, using administrative data to identify patients with cirrhosis can lead to misclassification.

of complications (decompensated cirrhosis) is often long, with a reported median survival of 12 years. ${ }^{5}{ }^{6}$ Compared with age-matched patients without cirrhosis, patients with cirrhosis have worse health and more comorbid conditions, resulting in greater use of healthcare services, including more hospital visits, nursing home stays and physician visits. ${ }^{2}$

Chronic hepatitis $\mathrm{C}$ virus infection is one of the main causes of cirrhosis and it is the most common indication for liver transplantation in Spain and worldwide. ${ }^{78}$ Hepatitis $\mathrm{C}$ infection affects the liver, and is associated with hypertension and other cardiovascular diseases, chronic renal impairment and diabetes mellitus. Some studies have estimated the costs of hepatitis $\mathrm{C}$ infection without, however, analysing the impact of complications of advanced liver disease. ${ }^{4}$

Knowing the impact of decompensated disease on outcomes and on the cost of treating advanced liver disease is important 
for planning resource allocation and preventive strategies, especially in a public health system. ${ }^{8}$ Because of its importance in public health, a growing number of publications address chronic liver disease..$^{2-11}$

This study aimed to describe overall healthcare resource use and expenditures in a non-selected population of patients with cirrhosis stratified into those with compensated disease and those with decompensated disease.

\section{MATERIALS AND METHODS}

Data source and study design

We analysed healthcare resource use and expenditures in 2013 in patients with cirrhosis residing in Catalonia, an autonomous region in North-East Spain with 7553650 inhabitants (density, 232.8 inhabitants $/ \mathrm{km}^{2}$ ). The regional health department, named CatSalut, provides universal healthcare coverage to all residents and collects detailed information on healthcare usage, including information from the minimum basic data set registered by healthcare units (eg, hospitals, primary care centres, nursing facilities and mental health centres). CatSalut also collects information on drug prescription and billing for services (eg, outpatient visits to specialists, emergency department visits, non-urgent medical transportation, outpatient rehabilitation, home oxygen therapy and dialysis). Initially, the Catalan health administration deployed a series of registers to record healthcare units' activity. In 2011, the Catalan Health Surveillance System (CHSS) was created to integrate most of those activity registers, placing the patient (instead of the provider) in the centre of this information system, thus favouring longitudinal analysis and providing a more holistic and transversal view of health problems. CHSS includes all the diagnoses reported by the different providers, regardless of whether they were recorded as the primary or secondary diagnosis. This information system collects all information from the entire public health system, including all hospital admissions and healthcare visits. CHSS contains 529 million diagnoses, 440 million contacts with various public health services and 519 million prescriptions dispensed by pharmacies. Its automated data validation system checks the consistency of the data and identifies potential errors. Moreover, as this information is used for healthcare provider payment purposes, periodic external audits are carried out to ensure the quality and reliability of the data.

Information from private health centres was not available for analysis, because although private hospitals notify CatSalut of their activity, they do not associate the reported data with each patient's identification number. Therefore, CatSalut has information about the activity performed, but not for individual patients. CatSalut administrative data showed that in 2013, of 2878 unplanned hospitalisations of patients with cirrhosis, $2727(94.8 \%)$ took place in public hospitals and only 151 $(5.2 \%)$ in private hospitals.

\section{Selection of patients}

This retrospective fixed cohort study included all residents of Catalonia aged 18 years or older diagnosed with cirrhosis on or before 31 December 2012 who were alive on that date. The International Classification of Diseases, ninth revision, Clinical Modification codes were used to identify cases with cirrhosis (codes 571.2 and 571.5) (see online supplementary appendix 1) as well as complications of cirrhosis.

We defined hepatic decompensation as an unscheduled hospital admission for hepatic encephalopathy, ascites, spontaneous bacterial peritonitis, hepatorenal syndrome, or hepatic complications such as hepatocellular carcinoma, oesophageal varices or portal hypertension. We divided patients into three groups:

- Compensated group: patients with cirrhosis who had not had any episode of hepatic decompensation before 2013.

- Previous decompensation group: patients who had had at least one episode of hepatic decompensation before 2012 but none during 2012.

- Recent decompensation group: patients who had at least one episode of hepatic decompensation during 2012.

\section{Calculation of expenditure}

The primary outcome variable of the study was expenditures related to healthcare resource use in 2013. In Catalonia, expenditures for healthcare and pharmacy services are normally directly attributed to each patient through their personal health identification number. Expenditures for primary care are calculated indirectly from a standard price per visit weighted by the attending professional (physician or nurse) and site of assistance. Expenditures for hospital care are weighted by diagnosis-related groups. Expenditures for inpatient care at hospitals, skilled nursing facilities and mental health centres are calculated according to length of stay (online supplementary table $1 \mathrm{~s}$ shows the average price by activity in Catalonia). The costs are attributed to each person through their personal identification codes assigned by the public health system.

The prices used in the calculations are the rates that CatSalut pays healthcare providers, which are published annually in the Official Gazette of the Government of Catalonia.

CatSalut's budget for 2013 was $€ 8085$ million, of which $€ 7885$ million ( $97.5 \%$ ) was devoted to healthcare services. Emergency medical transport and screening for breast and colon cancers accounted for the largest proportion of expenditures that were not individually allocated. The healthcare services in CatSalut's morbidity database accounted for $€ 7502$ million (€968 per capita), which represents $95.1 \%$ of CatSalut's expenditure on health services.

In this population-based study, the methods used allowed us to analyse the expenditures on patients with cirrhosis, but not the costs attributable only to the 
Table 1 Demographic characteristics according to liver disease group

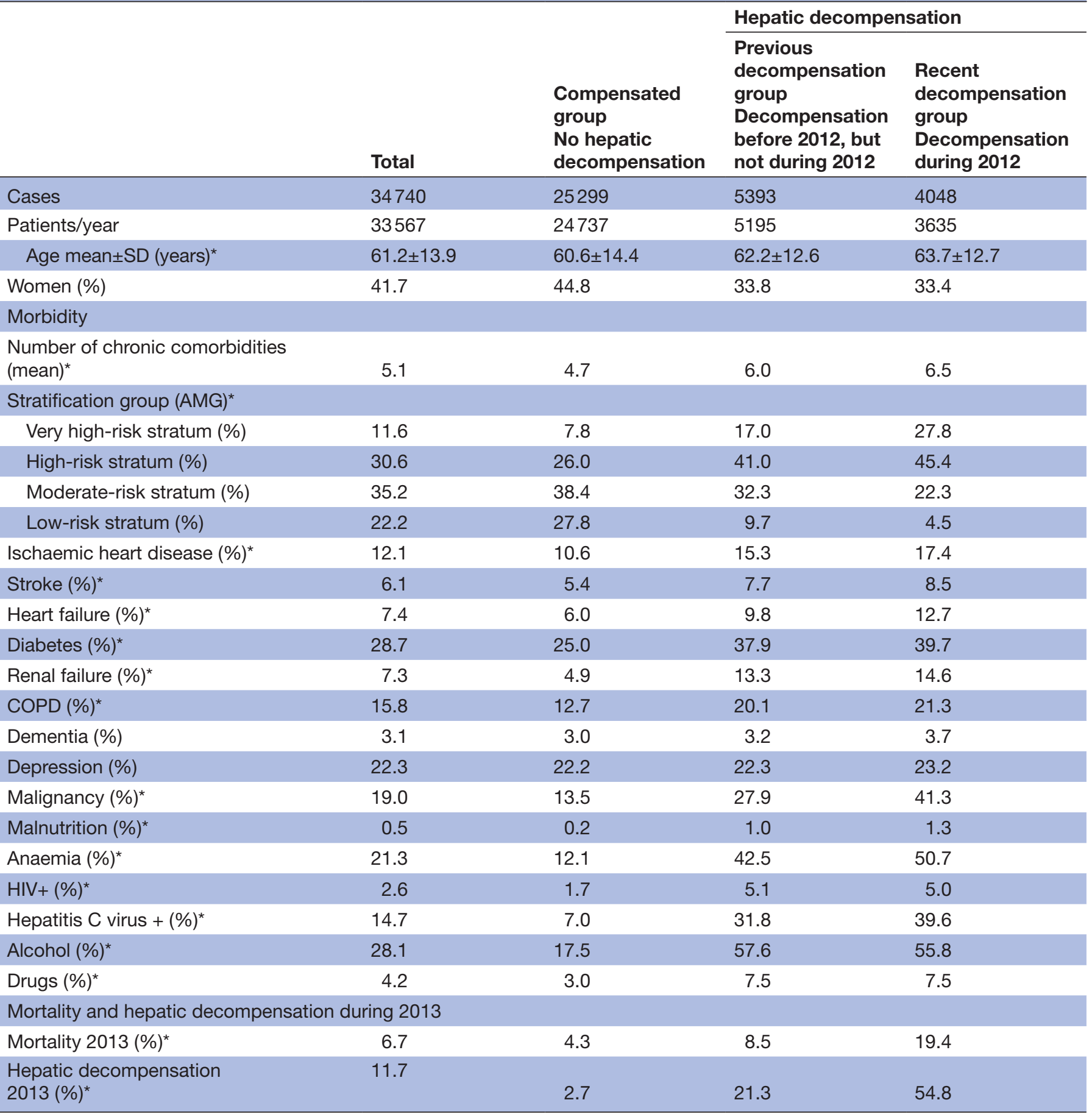

${ }^{*} P<0.001$

AMG, Adjusted Morbidity Grouper; COPD, chronic obstructive pulmonary disease.

disease itself. Thus, we calculated the total healthcare expenditure per person per year (in euros) for patients with cirrhosis (including cirrhosis-related care and care related to comorbidities), rather than the expenditure specifically associated with cirrhosis care. This approach enabled a comprehensive analysis of healthcare expenditures in this group of complex patients. To account for shorter follow-up periods due to deaths, time at risk was calculated in days from 31 December 2012 to the date of death (or 365 days otherwise) and transformed to years. Thus, the unit of analysis is not the patient but rather the patient/year.

\section{Assessment of predictors of increased expenditure}

We also sought to identify independent predictors associated with yearly expenditures greater than or equal to the 85 th centile of the distribution in the study population. This analysis included only patients who survived 
Table 2 Health services utilisation in 2013 in function of liver disease group

\begin{tabular}{|c|c|c|c|}
\hline & \multirow[b]{2}{*}{$\begin{array}{l}\text { Compensated group } \\
\text { No hepatic } \\
\text { decompensation }\end{array}$} & \multicolumn{2}{|l|}{ Hepatic decompensation } \\
\hline & & $\begin{array}{l}\text { Previous decompensation } \\
\text { group } \\
\text { Decompensation before } \\
\text { 2012, but not during } 2012\end{array}$ & $\begin{array}{l}\text { Recent decompensation } \\
\text { group } \\
\text { Decompensation during } \\
2012\end{array}$ \\
\hline Cases & 25299 & 5393 & 4048 \\
\hline Admission rate $(\times 100)^{*}$ & 29.2 & 60.8 & 117.9 \\
\hline Unplanned admission rate $(\times 100)^{*}$ & 16.5 & 37.8 & 78.3 \\
\hline Patients with unplanned admission (\%) ${ }^{\star}$ & 10.8 & 22.9 & 40.3 \\
\hline Patients $>1$ unplanned admission $(\%)^{\star}$ & 3.4 & 8.4 & 18.5 \\
\hline Emergency visit rates $(\times 100)^{\star}$ & 71.2 & 111.1 & 186.8 \\
\hline Other hospital outpatient visits (average) & 3.1 & 4.3 & 4.8 \\
\hline Primary care visits (average) ${ }^{\star}$ & 11.8 & 13.4 & 16.5 \\
\hline Users of a skilled nursing facility (\%)* & 4.0 & 7.3 & 15.7 \\
\hline Mental health outpatient visits rate $(\times 100)$ & 16.6 & 13.5 & 15.1 \\
\hline Psychiatric hospital admission rate $(\times 100)$ & 0.6 & 0.7 & 1.6 \\
\hline
\end{tabular}

${ }^{*} \mathrm{P}<0.001$

Likewise, the mean length of hospitalisation increased from 1.8 days in compensated patients to 4.5 days in previously decompensated patients to 9.6 days in recently decompensated patients.

the whole study period $(\mathrm{n}=32414)$. Predictors assessed were age, sex, comorbidities included in the Charlson Index, ${ }^{12}{ }^{13}$ previous healthcare utilisation and a novel population-based health risk assessment tool deployed in Catalonia, the Adjusted Morbidity Grouper (AMG), which is used to calculate an individual's morbidity burden. ${ }^{14}$ AMG categorises each patient in a risk-stratification pyramid with five strata:

- Basal-risk stratum: comprising individuals with minimum morbidity burden; $50 \%$ of individuals in the overall population of Catalonia (but $0 \%$ of patients with cirrhosis) fall into this stratum.

- Low-risk stratum: comprising individuals with low level complexity; $30 \%$ of individuals in the overall population of Catalonia (but 22.2\% of patients with cirrhosis) fall into this stratum.

- Moderate-risk stratum: comprising individuals with higher complexity than the previous risk stratum; $15 \%$ of individuals in the overall population of Catalonia (but $35.6 \%$ of patients with cirrhosis) fall into this stratum.

- High-risk stratum: comprising individuals with a greater morbidity burden than the previous stratum; $4 \%$ of individuals in the overall population of Catalonia (but $30.6 \%$ of patients with cirrhosis) fall into this stratum.

- Very high-risk stratum: comprising individuals with the highest morbidity burden; $1 \%$ of individuals in the overall population of Catalonia (but $11.6 \%$ of patients with cirrhosis) fall into this stratum.

\section{Statistical analysis}

Continuous variables are reported as means \pm SDs. For the univariate analysis, we used $\chi^{2}$ tests to compare categorical variables and analysis of variance to compare continuous variables. To identify independent predictors of increased healthcare expenditure, we used Multivariate logistic regression. Variables were entered in the model one by one and retained when their significance was $<0.10$. To evaluate the discriminatory ability of the resulting predictive model for identifying patients with cirrhosis with healthcare expenditures $\geq 85$ th centile, we calculated the area under the receiver operating characteristic (ROC) curve. ${ }^{15}$ Goodness of fit of the model was evaluated using the Hosmer-Lemeshow method. Statistical analyses were performed using SPSS software, V.18.0. All statistical tests and CIs were constructed with a type I error level of $5 \%$ and $\mathrm{P}$ values $<0.05$ were considered statistically significant.

\section{Ethics}

The study used retrospective data from administrative databases and patients were anonymous to the researchers. Our ethics committee stated that under Spanish legislation informed consent and ethics committee approval were not required. 

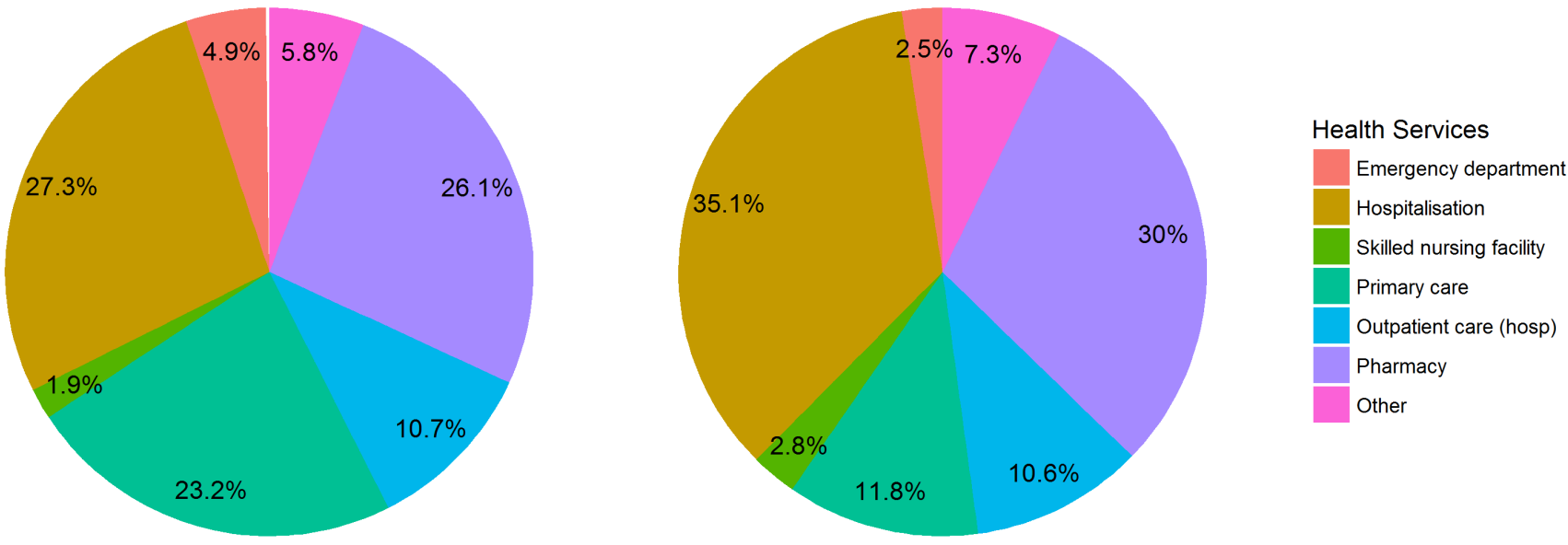

Figure 1 Health expenditure by type of resource in the general population and in patients with cirrhosis, in millions of euros. The category 'Other' includes mental health services, non-urgent medical transportation, outpatient rehabilitation and home oxygen therapy.

\section{RESULTS}

\section{Demographics of included patients}

We documented 34740 patients in Catalonia with cirrhosis on 31 December 2012 (460 per 100000 inhabitants); of these 25299 (72.8\%) had never had any episode of hepatic decompensation (compensated group), $5393(15.5 \%)$ had had at least one episode of decompensation before 2012 (previous decompensation group) and $4048(11.7 \%)$ had at least one episode of decompensation during 2012 (recent decompensation group). In the entire group, mean age was $61.2 \pm 14$ years and $41.8 \%$ were women; however, the proportion of women decreased with worsening disease $(44.8 \%$ in the compensated group, $33.8 \%$ in the previous decompensation group and $33.4 \%$ in the recent decompensation group) $(\mathrm{P}<0.001)$.

Patients with advanced liver disease had more comorbidities and their comorbidities were more severe than those with less advanced disease. There were no significant differences in dementia or depression between groups (table 1; see also online supplementary table $2 \mathrm{~s}$ ).

Hepatitis $\mathrm{C}$ infection was more common in patients who had episodes of decompensation, as was the use of sedatives and alcohol and/or drug abuse.

Overall mortality during 2013 was $6.7 \%$. Mortality was $4.3 \%$ in the compensated group, $8.5 \%$ in the previous decompensation group and $19.4 \%$ in the recent decompensation group.

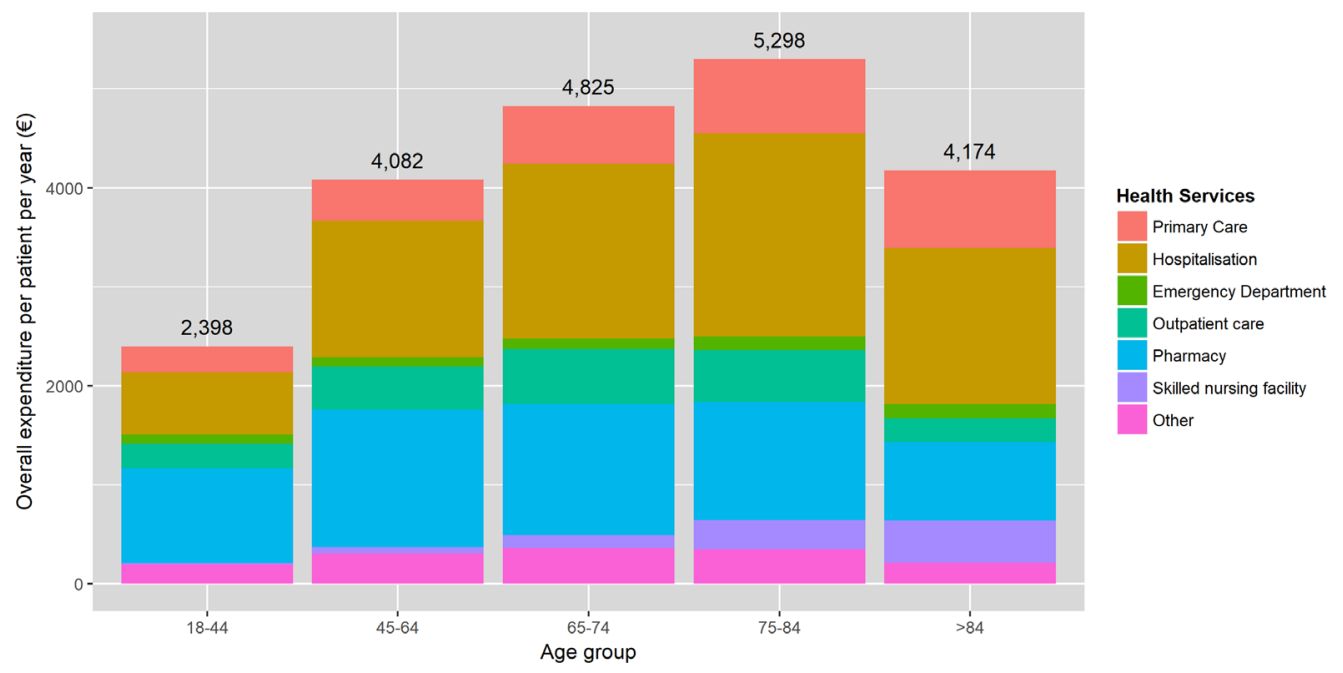

Figure 2 Healthcare resource use in patients with cirrhosis by age. 


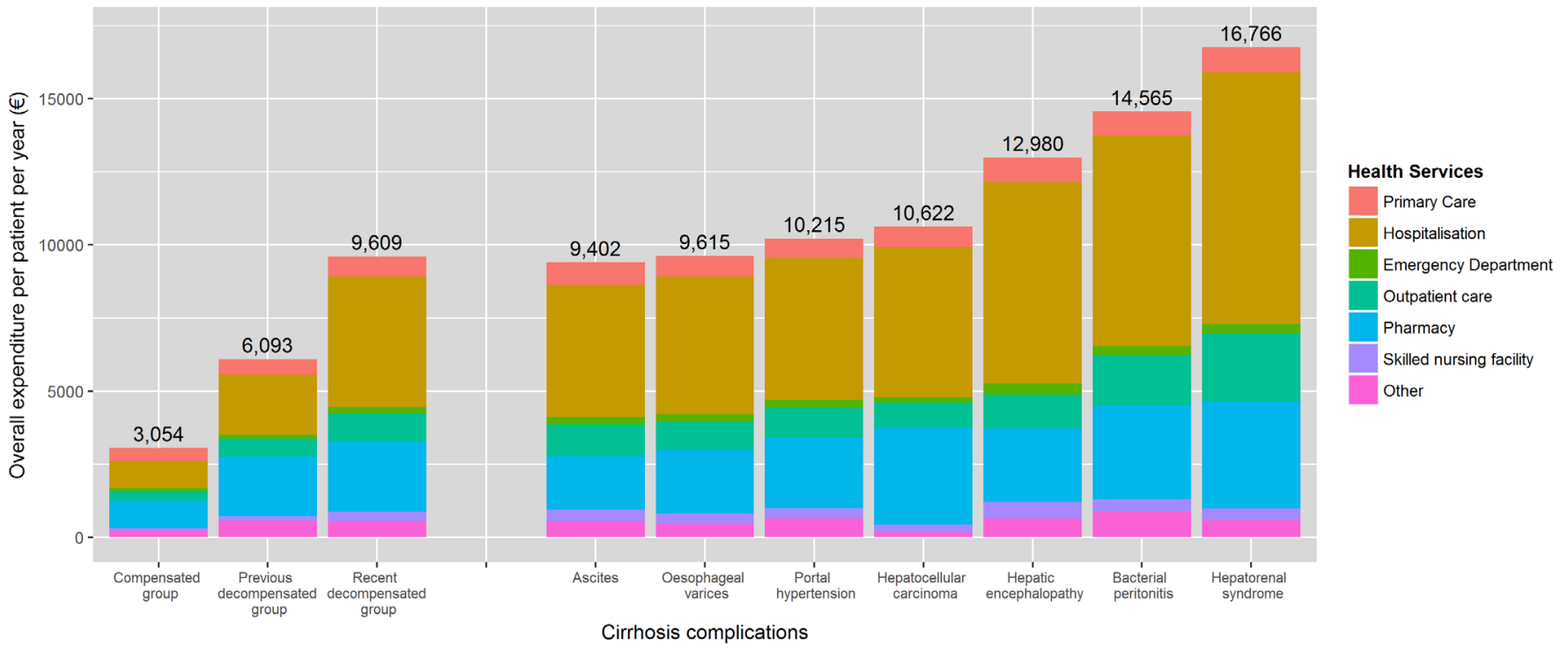

Figure 3 Healthcare resource use in patients with cirrhosis by hepatic decompensation.

Online supplementary table 3s shows these patients' probability of dying, adjusted for different factors.

\section{Use of health resources}

The overall rate of hospitalisations was 44.4 per 100 patients; the rate increased with severity $(29.2$ in the compensated group, 60.8 in the previous decompensation group and 117.9 in the recent decompensation group; $\mathrm{P}<0.0001$ ) (table 2 ). Online supplementary table $4 \mathrm{~s}$ shows the probability of having at least one urgent hospital admission, adjusted by different factors.

The rate of hospital admissions from the emergency room in the entire group was 27 per 100 patients, being
16.5 in the compensated group, 37.8 in the previous decompensation group and 78.3 in the recent decompensation group $(\mathrm{P}<0.0001)$. Whereas $33.8 \%$ of the patients in the compensated group presented at the emergency department at least once and $15.7 \%$ more than once, $45.5 \%$ of those in the previous decompensation group presented at least once and $25.7 \%$ more than once and $61.4 \%$ of those in the recent decompensation group presented at least once and $38.8 \%$ more than once (table 2 and online supplementary table $5 \mathrm{~s}$ ).

Similarly, the number of gastroenterology outpatient visits significantly increased with hepatic decompensation. The number of outpatient visits to other departments

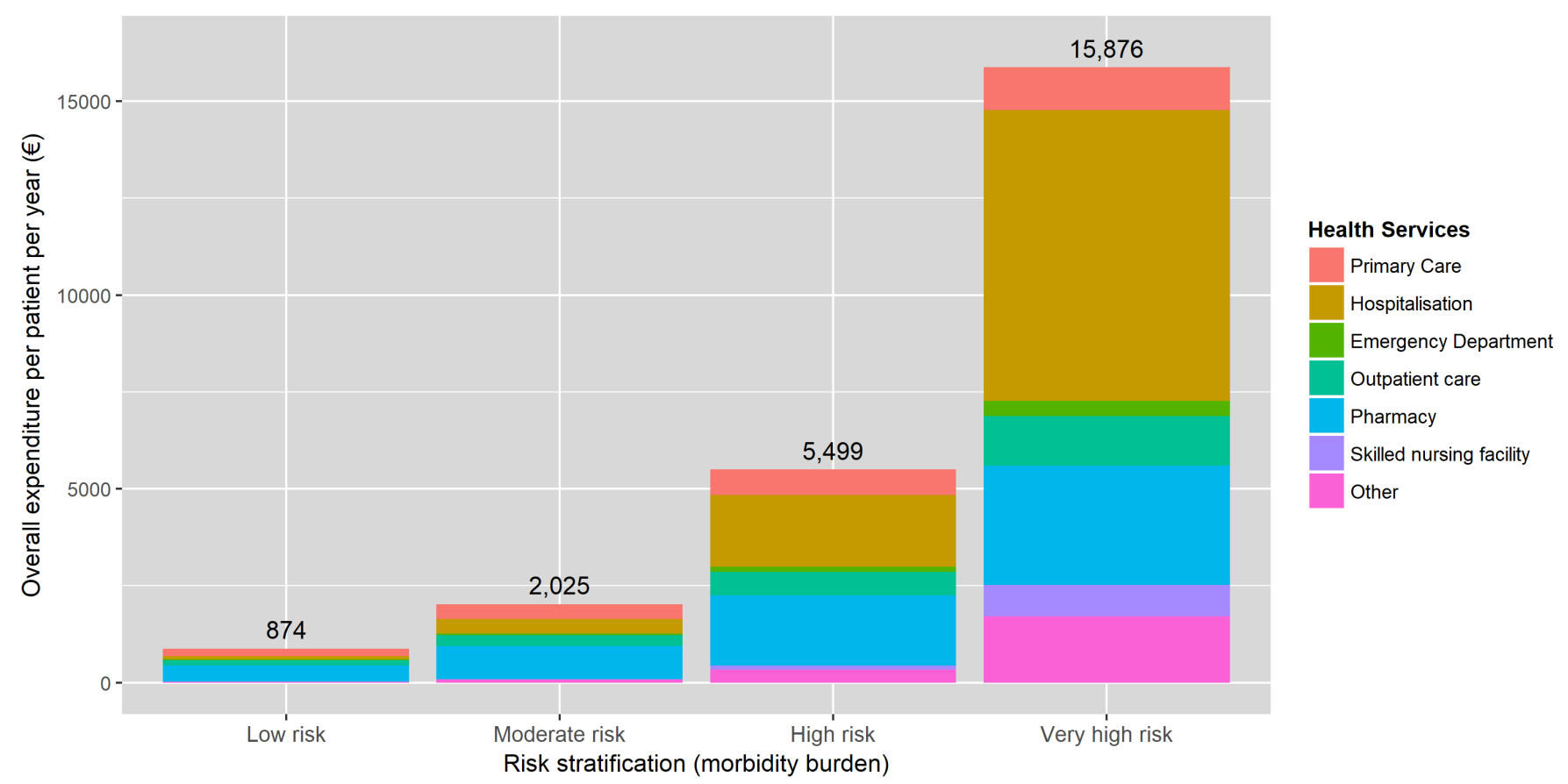

Figure 4 Healthcare resource use in patients with cirrhosis by risk stratification (morbidity burden). 
Table 3 Multivariate logistic regression analysing factors associated with expenditure higher than the 85th percentile

n $\quad$ c coefficient OR $95 \% \mathrm{Cl}$

\begin{tabular}{|c|c|c|c|c|c|}
\hline \multicolumn{6}{|l|}{ Sex } \\
\hline Male & 18783 & & 1 & - & - \\
\hline Female & 13631 & -0.034 & 0.967 & 0.890 & 1.050 \\
\hline \multicolumn{6}{|l|}{ Age group } \\
\hline 18-44 years old & 4152 & & 1 & - & - \\
\hline 45-64 years old & 15027 & 0.037 & 1.037 & 0.881 & 1.222 \\
\hline $65-74$ years old & 7540 & -0.154 & 0.858 & 0.720 & 1.022 \\
\hline $75-84$ years old & 4750 & -0.515 & 0.597 & 0.496 & 0.720 \\
\hline$>84$ years old & 945 & -1.209 & 0.299 & 0.225 & 0.396 \\
\hline \multicolumn{6}{|l|}{ Hepatic decompensation } \\
\hline Compensated group & 24219 & & 1 & - & - \\
\hline Previous decompensation group & 4934 & 0.311 & 1.364 & 1.230 & 1.514 \\
\hline Recent decompensation group & 3261 & 0.687 & 1.988 & 1.770 & 2.234 \\
\hline \multicolumn{6}{|l|}{ Risk stratum (morbidity burden) } \\
\hline Low-risk stratum & 7694 & & 1 & - & - \\
\hline Moderate-risk stratum & 12202 & 1.126 & 3.082 & 2.454 & 3.870 \\
\hline High-risk stratum & 9758 & 2.769 & 15.944 & 12.780 & 19.891 \\
\hline Very high-risk stratum & 2760 & 4.850 & 127.704 & 100.533 & 162.218 \\
\hline Chronic kidney disease & 2075 & 0.113 & 1.120 & 0.988 & 1.269 \\
\hline Hepatitis C virus + & 4377 & 0.429 & 1.536 & 1.389 & 1.699 \\
\hline $\mathrm{HIV}+$ & 823 & 2.930 & 18.734 & 15.374 & 22.828 \\
\hline \multicolumn{6}{|c|}{ Unplanned hospitalisation during 2012} \\
\hline 0 & 27687 & & 1 & - & - \\
\hline 1 & 3208 & 0.117 & 1.124 & 0.992 & 1.274 \\
\hline 2 & 944 & 0.168 & 1.183 & 0.974 & 1.437 \\
\hline 3 & 326 & 0.461 & 1.586 & 1.176 & 2.139 \\
\hline$>3$ & 249 & 0.507 & 1.660 & 1.166 & 2.363 \\
\hline \multicolumn{6}{|c|}{ Emergency department visits during 2012} \\
\hline 0 & 20034 & & 1 & - & - \\
\hline $1-2$ & 8919 & 0.190 & 1.209 & 1.095 & 1.334 \\
\hline $3-5$ & 2648 & 0.415 & 1.514 & 1.314 & 1.743 \\
\hline$>5$ & 813 & 0.580 & 1.785 & 1.431 & 2.226 \\
\hline Skilled nursing facility during 2012 & 820 & -0.339 & 0.713 & 0.587 & 0.865 \\
\hline Constant & & -4.593 & 0.010 & - & - \\
\hline
\end{tabular}

also increased, but the difference did not reach statistical significance.

All patients had frequent contact with primary care physicians and/or nurses; the number of primary care visits increased with decompensation (11.8 in the compensated group, 13.4 in the previous decompensation group and 16.5 in the recent decompensation group; $\mathrm{P}<0.0001)$. The rate of outpatient visits to mental health centres and the rate of psychiatric hospitalisation did not differ among groups. Moreover, the sicker the patients were, the more they required social services from convalescence centres, intermediate-stay or long-stay centres and palliative care centres.

\section{Assessment of healthcare expenditure}

During 2013, a total of $€ 142.1$ million was spent in the care of patients with cirrhosis in Catalonia $(1.8 \%$ of the total 2013 healthcare budget), representing an average expenditure of $€ 4234$ per patient/year.

Figure 1 displays the distribution of healthcare-related expenditures in patients with cirrhosis and in the general population of Catalonia. In patients with cirrhosis, the main source of expenditure was hospitalisation (for all causes), which accounted for $35.1 \%$ of 
the total; pharmacy costs accounted for $30 \%$, primary care for $11.8 \%$ and hospital outpatient care for $10.6 \%$. In contrast, in the general population the main sources of expenses were hospitalisation (27.3\%), pharmacy $(26.1 \%)$, primary care $(23.2 \%)$ and hospital outpatient care $(10.7 \%)$.

Figure 2 shows the distribution of healthcare-related expenditures inpatients with cirrhosis, stratified by age group. Despite some differences in resource use and expenditure among the different age groups, hospitalisation accounted for the greatest expenditure in all groups.

Total medical expenditure was significantly higher for patients in the recent decompensation group than for those in the previous decompensation and compensated groups. In the recent decompensation group, hospitalisation was the main expenditure, accounting for more than half of all costs (figure 3). By contrast, the total expenditure in the compensated group was divided nearly equally among pharmacy, hospitalisation and outpatient care (hospital and primary care). The distribution of expenditures in the previous decompensation group lay between those of the other two groups. Figure 3 also shows the distribution of expenditures in 2013 in patients in the recent decompensation group according to the type of hepatic decompensation occurring in 2012; hepatorenal syndrome, followed by spontaneous bacterial peritonitis and hepatic encephalopathy, generated much higher expenditures than other complications, mainly due to hospitalisation.

In patients with cirrhosis, the average healthcare expenditure increased with the number of chronic comorbidities, from $€ 773 /$ year in patients with one comorbidity to $€ 14,853$ /year in those with more than nine comorbidities. The AMG stratum was higher in patients with more comorbidities and expenditures increased exponentially with increasing AMG strata, from $<€ 900$ for patients in the low-risk stratum to $>€ 15000$ for those in the very high-risk stratum (figure 4).

In patients with lower healthcare resource use (with expenditures $<85$ th centile), expenditures were nearly equally distributed among primary care, pharmacy and hospitalisation. In patients with the highest healthcare resource use (expenditures $\geq 85$ th percentile), hospitalisation accounted for nearly half of all expenditures; total healthcare expenditure in this group was 10 times higher (€17822 per patient/year vs $€ 1806$ per patient/year) than in patients with lower resource use.

Table 3 reports the Multivariate logistic regression analysis to identify predictors of high expenditure. Morbidity burden (AMG stratum), HIV infection, hospitalisation and emergency room visits during 2012 were associated with expenditures $\geq 85$ th centile (ie, $>€ 7275$ per patient). The area under the ROC curve for identifying patients with expenditures $\geq 85$ th centile was 0.888 (95\% CI 0.883 to 0.893 , Hosmer-Lemeshow test: $\left.\mathrm{X}^{2}=29.997 \quad(\mathrm{P}<0.001)\right)$. These results show that the model identifies which patients will have higher health expenditures.

\section{DISCUSSION}

In our study, liver cirrhosis had an important impact on healthcare expenditures, and hospitalisation and pharmacy accounted for the largest proportion of costs associated with treating patients with cirrhosis. To our knowledge, this is the first population-based study to quantify the total economic impact of cirrhosis in relation to hepatic decompensation and to determine the distribution of costs (including those related with primary, nursing, hospital and mental healthcare as well as physical therapy and pharmacy) in treating patients with this condition.

This population-based study conducted in Catalonia included around 7500000 million people, which is comparable to the population of some European countries.

In recent years, many studies have estimated the economic burden of hepatitis and cirrhosis ${ }^{16}$ because these conditions are associated with high morbidity, mortality and economic costs. Although one of these studies analysed the cost of care over the last year of life in patients with an end-stage liver disease, ${ }^{17}$ it was not a population-based study like ours. Another study described the direct costs related to hospital admissions in patients with cirrhosis. ${ }^{10}$ By contrast, our study included all healthcare-related expenses in patients with cirrhosis (during hospitalisation and afterwards). Another study analysed direct and indirect costs of chronic liver disease, but included all patients, not only those with cirrhosis. ${ }^{18}$

Liver cirrhosis leads to 800000 deaths every year, representing $1.3 \%$ of all deaths worldwide. According to WHO, cirrhosis is among the 18 most common causes of death. ${ }^{11}$ Five-year survival is $36 \%$ in alcoholic cirrhosis and $14 \%$ in non-alcoholic cirrhosis. ${ }^{11}$ We found that mortality increased significantly in patients who had a recent hepatic decompensation and the risk of mortality increased nearly $20 \%$ in patients with a decompensation in the previous 12 months.

The total cost of treating patients with cirrhosis in Catalonia in 2013 was almost $€ 150$ million (€4234 per patient). Hospitalisation remains the main source of healthcare-related expenditure, followed by medication, although hospital mortality has decreased in recent years. ${ }^{16}$ Patients with more advanced liver disease required more healthcare (more days hospitalised, emergency visits and primary care visits) and more medication, generating greater expenditures. In patients with cirrhosis, the probability of dying within a year of a hepatic decompensation is $34 \% .{ }^{19}$ In our study, mortality was nearly 2.3 times higher in patients with a recent decompensation than in patients who had gone at least a year since a prior decompensation and 4.5 times higher than those who had never had a decompensation. Our model's ability to differentiate patients who will require high expenditures suggests that our study can be useful in health planning, resource allocation and evaluation of results.

Our large database allowed us to calculate the morbidity burden using $\mathrm{AMG}^{20}$ and to stratify the risk of morbimortality. In our population, morbidities were common and 
greater severity of liver disease was associated with higher morbidity burden $(>75 \%$ of all patients were in the moderate-risk or higher-risk strata: $72.2 \%$ in the compensated group, $90.3 \%$ in the previous decompensation group and $95.5 \%$ in the recent decompensation group). Moreover, expenditures increased with increasing AMG strata, so controlling comorbidities is one way to reduce costs.

Another way to reduce costs is to modify the natural history of the liver disease. Studies that have analysed the direct and indirect costs of treating hepatitis $\mathrm{C}$ infection point out that costs rise with the natural history of the disease. ${ }^{172122}$ New antiviral agents against hepatitis $C$ have modified the natural history of the disease, but the impact of these agents on the overall cost of treating patients with hepatitis $\mathrm{C}$ infection will not become clear until a few years have passed. ${ }^{23}$ Nevertheless, our results suggest that this impact is likely to be significant because expenditures in patients with recent hepatic decompensation totalled more than twice those in patients who had not had a decompensation for at least 1 year and more than three times those in patients with compensated cirrhosis.

Although some studies have analysed the costs of caring for other diseases such as diabetes mellitus ${ }^{24}$ or heart failure, ${ }^{20}$ to our knowledge, this is the first population-based study to analyse morbidity and costs in patients with liver disease taking into account hepatic decompensation. Although several studies have evaluated some aspects involved in caring for patients with cirrhosis (hospitalisation, caregiving hours), ${ }^{2}$ our study analysed overall costs, including expenditures on nursing, primary care and mental health.

This study has some limitations. The use of a general population database minimised selection bias and allowed us to analyse a real-world population. However, using administrative data to identify patients with cirrhosis can lead to misclassification, especially in patients diagnosed in private centres who have not used public resources.

Moreover, this administrative database did not include important information such as the aetiology of cirrhosis (although alcohol consumption and/or hepatitis C infection are recorded in the database) or other important clinical data for characterising and understanding the study population and the evolution of the disease. For these reasons, we cannot calculate classical scores such as Model For End-Stage Liver Disease (MELD), Child-Pugh or Acute-on-chronic Liver Failure (ACLF).

Patients' need for social services increases when liver disease becomes decompensated. The costs associated with this increase are, however, difficult to quantify and vary widely among health systems. Although these data are provided in the supplementary data, caution is warranted in interpreting these results.

Finally, we excluded patients who died during 2013 from the analysis of risk factors, although they were included in the analysis of healthcare expenditures. The reason for this exclusion is that patients who died during the study period were excluded from the assessment of predictors of increased expenditure. Although this exclusion could lead to bias, the cost of these patients after their death was zero. Therefore, the inclusion of dead patients in the analysis could lead to a possible bias in the calculation of the ORs of risk factors and consequently to errors in interpretation when diseases with high mortality are analysed. Among the possible solutions for this bias, we opted for the simplest solution and we analysed only patients who remained alive throughout the year. This strategy has two main advantages: simplicity and robustness. In contrast to similar strategies, each of which introduces some sort of bias, we believe it is better to use the simplest and easiest strategy to explain and understand the data. Moreover, one of the strengths of our study is that as it is a population-based study with real data, there is no need for methodological sophistication when a simpler strategy can be used.

\section{CONCLUSIONS}

Cirrhosis results in high healthcare resource utilisation, leading to high expenditures. On 31 December 2012, the point prevalence in Catalonia was 459.9 per 100000 inhabitants. The cost of treating patients with cirrhosis was high because more than $75 \%$ had moderate to very high risk according to their AMG morbidity burden. The main expenditure was hospitalisation; its weight in the total expenditure increased with decompensation.

Avoiding excessive alcohol consumption and eradicating hepatitis $\mathrm{C}$ infection with the new antiviral agents will probably modify the natural history of cirrhosis, reducing morbidity and mortality and consequently expenditures in these patients.

Contributors All authors have contributed in the concept and design, $\mathrm{MC}$ and EV in data acquisition and statistical analysis, MM, MV, MC and EV in interpretation of data, and MM and MV in writing the manuscript. All authors have supervised and approved the manuscript.

Funding This research received no specific grant from any funding agency in the public, commercial or not-for-profit sectors.

Competing interests None declared.

Patient consent Not required.

Provenance and peer review Not commissioned; externally peer reviewed.

Data sharing statement Data are not included in this submission due to legal and privacy stipulations from the CatSalut (Catalan Health Department). Data are available to those completing the request for research identifiable files from the CatSalut upon acceptance by the Catalan Health Department. For contact, please reach the Analysis on Demand and Activity Division, Catalan Health Service.

Open Access This is an Open Access article distributed in accordance with the Creative Commons Attribution Non Commercial (CC BY-NC 4.0) license, which permits others to distribute, remix, adapt, build upon this work non-commercially, and license their derivative works on different terms, provided the original work is properly cited and the use is non-commercial. See: http://creativecommons.org/ licenses/by-nc/4.0/

(c) Article author(s) (or their employer(s) unless otherwise stated in the text of the article) 2018. All rights reserved. No commercial use is permitted unless otherwise expressly granted.

\section{REFERENCES}

1. Adams LA, Lymp JF, St Sauver J, et al. The natural history of nonalcoholic fatty liver disease: a population-based cohort study. Gastroenterology 2005;129:113-21. 
2. Rakoski MO, McCammon RJ, Piette JD, et al. Burden of cirrhosis on older Americans and their families: analysis of the health and retirement study. Hepatology 2012;55:184-91.

3. Sayiner M, Koenig A, Henry L, et al. Epidemiology of nonalcoholic fatty liver disease and nonalcoholic steatohepatitis in the united states and the rest of the world. Clin Liver Dis 2016;20:205-14.

4. Otgonsuren M, Henry L, Hunt S, et al. Resource utilization and survival among Medicare patients with advanced liver disease. Dig Dis Sci 2015;60:320-32.

5. Asrani SK, Kamath PS. Natural history of cirrhosis. Curr Gastroenterol Rep 2013;15:308.

6. Jafri SM, Gordon SC. The consequences of cirrhosis in America. Dig Dis Sci 2015;60:283-4.

7. García-Fulgueiras A, García-Pina R, Morant C, et al. Burden of disease related to hepatitis $C$ and hepatitis B in Spain: a methodological challenge of an unfolding health problem. $J$ Viral Hepat 2011;18:e453-460.

8. Mathers C, Stein C, Ma Fat D, et al. Global Burden of disease 2000: version 2 methods and results. Global programme on evidence for health policy discussion paper No 50. Geneva: World Health Organitzation, 2002. http://www.who.int/healthinfo/paper50.pdf

9. Chung W. The cost of liver disease in Korea: methodology, data and evidence. Clin Mol Hepatol 2015;21:14-21.

10. Vitor S, Marinho RT, Gíria J, et al. An observational study of the direct costs related to hospital admissions, mortality and premature death associated with liver disease in Portugal. BMC Res Notes 2016;9:62.

11. Akbari Sari A, Kazemi Karyani A, Alavian SM, et al. The Economic Burden of Liver Cirrhosis in Iran: a Cost of Illness Study. Iran J Public Health 2015;44:512-21.

12. Charlson ME, Pompei P, Ales KL, et al. A new method of classifying prognostic comorbidity in longitudinal studies: development and validation. J Chronic Dis 1987;40:373-83.

13. Deyo RA, Cherkin DC, Ciol MA. Adapting a clinical comorbidity index for use with ICD-9-CM administrative databases. J Clin Epidemiol 1992;45:613-9.
14. Dueñas-Espín I, Vela E, Pauws S, et al. Proposals for enhanced health risk assessment and stratification in an integrated care scenario. BMJ Open 2016;6:e010301.

15. Hanley JA, McNeil BJ. The meaning and use of the area under a receiver operating characteristic (ROC) curve. Radiology 1982;143:29-36.

16. Vergara $M$, Clèries $M$, Vela $E$, et al. Hospital mortality over time in patients with specific complications of cirrhosis. Liver Int 2013;33:828-33.

17. Gola A, Davis S, Greenslade L, et al. Economic analysis of costs for patients with end stage liver disease over the last year of life. BMJ Support Palliat Care 2015;5:110.2-110.

18. Stepanova M, De Avila L, Afendy M, et al. Direct and Indirect Economic Burden of Chronic Liver Disease in the United States. Clin Gastroenterol Hepatol 2017;15:759-66.

19. Solà R, Alvarez MA, Ballesté $B$, et al. Probability of liver cancer and survival in HCV-related or alcoholic-decompensated cirrhosis. A study of 377 patients. Liver Int 2006;26:62-72.

20. Farré $\mathrm{N}$, Vela $\mathrm{E}$, Clèries $\mathrm{M}$, et al. Medical resource use and expenditure in patients with chronic heart failure: a population-based analysis of 88195 patients. Eur J Heart Fail 2016;18:1132-40.

21. Gray E, O'Leary A, Kieran JA, et al. Direct costs of interferonbased and interferon-free direct-acting antiviral regimens for the treatment of chronic hepatitis $\mathrm{C}$ infection. J Viral Hepat 2016;23:677-86.

22. Martin NK, Vickerman P, Dore GJ, et al. Prioritization of HCV treatment in the direct-acting antiviral era: An economic evaluation. $J$ Hepatol 2016;65:17-25.

23. Manos MM, Darbinian J, Rubin J, et al. The effect of hepatitis C treatment response on medical costs: a longitudinal analysis in an integrated care setting. J Manag Care Pharm 2013;19:438-47.

24. American Diabetes Association. Economic costs of diabetes in the U.S. In 2007. Diabetes Care 2008;31:596-615. 